\title{
Distributed indeterminacy evaluation of cable-strut structures: formulations and applications"
}

\author{
Jin-yu ZHOU ${ }^{\dagger 1}$, Wu-jun $\mathrm{CHEN}^{1}$, Bing ZHAO ${ }^{2}$, Zhen-yu QIU ${ }^{2}$, Shi-lin DONG ${ }^{1,3}$ \\ ( ${ }^{1}$ Space Structures Research Center, Shanghai Jiao Tong University, Shanghai 200240, China) \\ $\left({ }^{2}\right.$ School of Naval Architecture, Ocean \& Civil Engineering, Shanghai Jiao Tong University, Shanghai 200240, China) \\ $\left({ }^{3}\right.$ College of Civil Engineering and Architecture, Zhejiang University, Hangzhou 310027, China) \\ †E-mail: zjysjtu@sjtu.edu.cn \\ Received Apr. 7, 2015; Revision accepted July 13, 2015; Crosschecked Aug. 7, 2015
}

\begin{abstract}
The indeterminacy evaluation is an effective method for system identification; it can predict the mechanical behaviors of flexible structures in the primary design. However, the conventional indeterminacy evaluation based solely on geometry and topology has neglected the influence of material properties on mechanical behavior and the contribution of each component to the total indeterminacy. To address these issues, a distributed indeterminacy evaluation taking account of the effect of component stiffness was carried out with a view to providing reasonable interpretations and feasible applications for two concepts, i.e., the distributed static indeterminacy (DSI) and the distributed kinematic indeterminacy (DKI). A unified method for the DSI is proposed, and a comparative analysis between this and an existing method revealed that the proposed method has a wider range of applicability and is essentially identical in the kinematically determinate case. It can be concluded that since the DSI is representative of symmetric properties, a simple but efficient grouping criterion can be established which can improve the efficiency of the specific force finding method entitled double singular value decomposition (DSVD). On the other side, an evaluable method for the DKI is proposed suggesting that DKI is a useful indicator for the assessment of nodal mobility and can provide a feasible solution to the form transforming study.
\end{abstract}

Key words: Flexible structures, Cable-strut structures, Distributed indeterminacy, Initial force design, Force finding, Singular value decomposition, Form transforming

doi: 10.1631 jzus.A1500081

Document code: A

CLC number: TU393.3

\section{Introduction}

Flexible structures with a diversity of structural forms, such as Geiger domes, Levy domes, Panta domes, and tensegrities, attract great attention from civil engineers to meet the design requirements of large-space, light-weight, and novel architectures (Eriksson and Tibert, 2006; Pai, 2011; Zhang et al., 2013). A crucial step of the structural design is the system identification, targeting a preliminary predic-

\footnotetext{
* Project supported by the National Natural Science Foundation of China (Nos. 51278299, 51478264, and 11172180)

(DD ORCID: Jin-yu ZHOU, http://orcid.org/0000-0001-6514-5081

(C) Zhejiang University and Springer-Verlag Berlin Heidelberg 2015
}

tion for the mechanical performance. If a structure is identified as a statically and/or kinematically indeterminate assembly, this implies that the mechanical behavior of this structure is highly relevant to selfstress states (Chen et al., 2014). Especially, the rigidity and the pre-stability of this structure is the result of a self-stress equilibrium between cables and struts (Ashwear and Eriksson, 2014).

It is widely accepted that for an entire assembly Maxwell's rule, the modified Maxwell's rule (Calladine, 1978) and the equilibrium matrix theory (Pellegrino and Calladine, 1986; Pellegrino, 1990; 1993; Calladine and Pellegrino, 1991) are fundamental to any understanding of its mechanical behavior. In fact, the conventional indeterminacy evaluation utilizing 
the concepts of static and the kinematic indeterminacy was derived from these basic theories and regarded as an effective method for system identification. However, this evaluation took no account of the influence of material properties on the mechanical behavior, as well as the contribution of each component to the total degree of indeterminacy.

To address these issues, another insight into system identification can be gained from a distributed indeterminacy evaluation that incorporates the effect of component stiffness. Little in the literature has focused on the theoretical studies regarding distributed indeterminacy. For example, Ströbel (1995) and Ströbel and Singer (2008) presented the concepts of elastic redundancy (ER) and geometric redundancy (GR) attempting to describe structural properties from the standpoint of each component, which is closely related to the distributed indeterminacy discussed in this work. Moreover, Tibert (2005a; 2005b) proposed an analytical method for distributed static indeterminacy (DSI), as the definition of the ER did not always conform to Maxwell's rule and the modified Maxwell's rule.

Despite the different definitions, these theoretical derivations had a common feature, i.e., the obtained matrixes are identical in the kinematically determinate case. It is known that this matrix is representative of the inherent properties of structures and can be used to evaluate structural sensitivity (Ströbel, 1995; Tibert, 2005a; 2005b; Ströbel and Singer, 2008; Jiang et al., 2012) and robustness (or vulnerability) (Kou et al., 2008; Chen et al., 2010; Chen and Kou, 2013; Gao and Liu, 2013). For sensitivity, this matrix was termed the redundancy matrix, while in the context of robustness it was referred to as the deformation matrix. By means of the deformation matrix, Chen et al. (2010) and Chen and Kou (2013) presented a transfer law and an importance coefficient of rigid joints that illuminated the redistribution of element forces or deformations produced by the removal of a certain element.

Although there emerged a few applications concerning the distributed indeterminacy, it has been found that the existing formula of the ER is limited to the kinematically determinate case, and the formula of the DSI seems ambiguous for lack of clear clarification. Additionally, a comparison between these formulas is indispensable to establish their inherent correlation, i.e., whether the results achieved by these methods are equivalent, or whether these methods are essentially identical. On the other side, little effort has been given to the concept complementary to the DSI, namely, the distributed kinematic indeterminacy (DKI).

In this paper, a distributed indeterminacy evaluation of flexible structures that incorporates the material properties is conducted systematically, including the static indeterminacy distribution and the kinematic indeterminacy distribution. The key objective of this work is to provide reasonable interpretations of the DSI and the DKI, and to develop their potential applications in engineering. To reach this objective, a unified method for the DSI that is valid in all cases is derived on the basis of equilibrium matrix theory, and then an evaluable method for the DKI is proposed for the first time to our knowledge. Finally, three examples are presented to verify the validity and accuracy of these theoretical derivations.

\section{Distributed static indeterminacy}

In this section, two analytical methods for the calculation of the DSI are provided: one will briefly be outlined for the provision of the definition, and the other will be adapted and improved from Tibert (2005a)'s study with necessary clarifications. In addition, these methods will be compared to reveal their inherent correlation.

\subsection{Mathematical formula for DSI}

2.1.1 A method based on minimum potential energy theory

The analytical method for the DSI originated by Ströbel (1995) was deduced on the basis of minimum potential energy theory; this whole derivation for the DSI was vitally concerned with the relationship between the element elongations and the element forces, while ignoring the effect of the external loads on the structural behaviors.

First, considering a 3D assembly with $n$ nodes, $b$ elements (struts and cables), and $c$ kinematic constraints, the total potential energy function $\Pi_{\mathrm{PE}}$ of the assembly can be established by (Ströbel, 1995): 


$$
\Pi_{\mathrm{PE}}=\underbrace{\frac{1}{2} \boldsymbol{e}^{\mathrm{T}} \boldsymbol{K} \boldsymbol{e}}_{\Pi_{i}}-\underbrace{\boldsymbol{t}^{\mathrm{T}}\left(\boldsymbol{l}_{0}+\boldsymbol{e}-\boldsymbol{l}\right)}_{\Pi_{a}}-\boldsymbol{f}^{\mathrm{T}} \boldsymbol{d},
$$

where $\boldsymbol{f}_{(3 n-c) \times 1}, \boldsymbol{t}_{b \times 1}, \boldsymbol{d}_{(3 n-c) \times 1}$, and $\boldsymbol{e}_{b \times 1}$ denote vectors of the external nodal loads, element forces, nodal displacements, and element elongations, respectively; $\Pi_{i}$ is the elastic strain energy and $\Pi_{a}$ is the external force potential energy; vector $\boldsymbol{l}_{0}$ is the initial element length and vector $\boldsymbol{l}$ is the current element length; matrix $\boldsymbol{K}=\operatorname{diag}\left(k_{1}, k_{2}, \ldots, k_{i}, \ldots, k_{b}\right)$ stands for the diagonal stiffness matrix, in which $k_{i}=a_{i} E_{i} / l_{i}$ is the axial stiffness of element $i$, with $a_{i}$ the cross section area, and $E_{i}$ the Young's modulus of elasticity.

Then, minimizing $\Pi_{\mathrm{PE}}$ with respect to different variables yields the equilibrium, compatibility, and constitutive equation, respectively:

$$
\begin{gathered}
A t=f \\
B d=e \\
t=K e
\end{gathered}
$$

where $\boldsymbol{A}_{(3 n-c) \times b}$ is the equilibrium matrix, and $\boldsymbol{B}_{b \times(3 n-c)}=\boldsymbol{A}^{\mathrm{T}}$ is the compatibility matrix.

For element elongations $\boldsymbol{e}$, it should contain two terms: one is caused by external nodal loads $f$ (Eq. (4)), and the other is the so-called initial elongation $\boldsymbol{e}_{0}$, i.e.,

$$
\boldsymbol{e}=\boldsymbol{e}_{0}+\boldsymbol{F t}
$$

where $\boldsymbol{F}=\boldsymbol{K}^{-1}$ is the diagonal flexibility matrix.

For the element forces $t$, a new expression can be obtained by substituting Eqs. (3) and (5) into Eq. (2):

$$
\boldsymbol{t}=\boldsymbol{K} \boldsymbol{A}^{\mathrm{T}}\left(\boldsymbol{A} \boldsymbol{K} \boldsymbol{A}^{\mathrm{T}}\right)^{-1} \boldsymbol{f}-\boldsymbol{K}\left[\boldsymbol{I}-\boldsymbol{A}^{\mathrm{T}}\left(\boldsymbol{A} \boldsymbol{K} \boldsymbol{A}^{\mathrm{T}}\right)^{-1} \boldsymbol{A} \boldsymbol{K}\right] \boldsymbol{e}_{0}=\boldsymbol{t}_{\mathrm{p}}+\boldsymbol{t}_{\boldsymbol{e}_{0}},(6)
$$

where $\boldsymbol{I}_{b \times b}$ stands for an identity matrix. The above equation demonstrates that the element forces $t$ can be divided into $\boldsymbol{t}_{\mathrm{p}}$ and $\boldsymbol{t}_{\boldsymbol{e}_{0}}$, where $\boldsymbol{t}_{\mathrm{p}}$ is generated by the external loads, and $\boldsymbol{t}_{\boldsymbol{e}_{0}}$ is caused by the initial elongations. Since the whole derivation ignores the effect of external loads, the element forces $t$ concerned herein can be expressed as:

$$
\boldsymbol{t}=\boldsymbol{t}_{e_{0}}=-\boldsymbol{K} \Lambda \boldsymbol{e}_{0}
$$

where

$$
\boldsymbol{\Lambda}=\boldsymbol{I}-\boldsymbol{A}^{\mathrm{T}}\left(\boldsymbol{A} \boldsymbol{K} \boldsymbol{A}^{\mathrm{T}}\right)^{-1} \boldsymbol{A} \boldsymbol{K},
$$

which is termed the redundancy matrix or the deformation matrix. It is seen from Eq. (7) that matrix $\boldsymbol{K} \boldsymbol{\Lambda}$ acts as a bridge between the change of element forces $\boldsymbol{t}$ and the input of the initial elongations $\boldsymbol{e}_{0}$. As the element value of matrix $\boldsymbol{K}$ is constant on a given stiffness assignment, matrix $\Lambda$ can be treated as a scaling matrix and then its diagonal entry $\gamma_{i}(i=1$, $2, \ldots, b)$ is defined as the DSI.

It is found that because of the idempotent matrix $\Lambda$, the range of each DSI is between zero and one, and the sum of DSIs is equal to the total degree of static indeterminacy. This finding implies that the DSI can reflect the contribution of each element to the static indeterminacy. Nevertheless, the applicability of this formula is limited to the kinematically determinate case, due to the singularity of matrix $\boldsymbol{A} \boldsymbol{K} \boldsymbol{A}^{\mathrm{T}}$ in the other case.

2.1.2 A unified method based on equilibrium matrix theory

To avoid this limitation, a unified method for the DSI will be adapted from Tibert (2005a)'s study and revised with necessary clarifications. Based on equilibrium matrix theory, the unified method intends to extend the range of applicability to include the kinematically indeterminate assemblies. At the start of this derivation, the equibrium matrix $\boldsymbol{A}$ is decribed by the singular value decomposition (SVD) as

$$
\boldsymbol{A}=\boldsymbol{U}\left[\begin{array}{cc}
\boldsymbol{R}_{1} & \mathbf{0} \\
\mathbf{0} & \mathbf{0}
\end{array}\right] \boldsymbol{V}^{\mathrm{T}},
$$

where matrix $\boldsymbol{R}_{1}=\operatorname{diag}\left(\mu_{1}, \mu_{2}, \ldots, \mu_{r_{A}}\right)$ in which $r_{\boldsymbol{A}}$ is the rank and $\mu$ is the non-zero singular value of ma$\operatorname{trix} \boldsymbol{A} ; \boldsymbol{U}\left(\in \mathbb{R}^{(3 n-c) \times(3 n-c)}\right)$ is the left orthogonal matrix, and $\boldsymbol{V}\left(\in \mathbb{R}^{b \times b}\right)$ is the right orthogonal matrix. Both orthogonal matrices are implicitly normalized in this process, and can be partitioned into two submatrices:

$$
\begin{aligned}
& \boldsymbol{V}=\left[\boldsymbol{v}_{1}, \boldsymbol{v}_{2}, \ldots, \boldsymbol{v}_{r_{A}} \mid \boldsymbol{v}_{r_{A}+1}, \ldots, \boldsymbol{v}_{3 n-c}\right]=\left[\boldsymbol{V}_{r_{A}} \mid \boldsymbol{S}\right], \\
& \boldsymbol{U}=\left[\boldsymbol{u}_{1}, \boldsymbol{u}_{2}, \ldots, \boldsymbol{u}_{r_{A}} \mid \boldsymbol{u}_{r_{A}+1}, \ldots, \boldsymbol{u}_{b}\right]=\left[\boldsymbol{U}_{r_{A}} \mid \boldsymbol{M}\right],
\end{aligned}
$$


where matrix $\boldsymbol{S}\left(\in \mathbb{R}^{b \times s}\right)$ collects the basic bases for self-stress modes and $s=b-r_{A}$ is the total degree of static indeterminacy; matrix $\boldsymbol{M}\left(\in \mathbb{R}^{(3 n-c) \times m}\right)$ comprises the basic bases for mechanisms and $m=3 n-c-r_{A}$ is the total degree of kinematic indeterminacy.

Then, a general expression for element forces $t$ can be

$$
\boldsymbol{t}=\boldsymbol{A}^{+} \boldsymbol{f}+\boldsymbol{S} \alpha,
$$

where matrix $\boldsymbol{A}^{+}$stands for the generalized inverse of matrix $\boldsymbol{A}$, and $\boldsymbol{\alpha}=\left[\alpha_{1}, \alpha_{2}, \ldots, \alpha_{s}\right]^{\mathrm{T}}$ is the coefficient vector of $s$ independent self-stress modes.

For a statically indeterminate assembly, a specific vector $\boldsymbol{\alpha}$ can be determined by a set of $s$ compatibility equations. As the element elongations $\boldsymbol{e}$ are composed of two orthogonal parts, i.e., the compatible and the incompatible elongations (Pellegrino and Calladine, 1986), when not considering the effect of external loads these compatibility equations can be written as

$$
\boldsymbol{S}^{\mathrm{T}}\left[-\boldsymbol{e}_{0}-\boldsymbol{F S} \boldsymbol{\alpha}\right]=\mathbf{0},
$$

therefore

$$
\boldsymbol{\alpha}=-\left(\boldsymbol{S}^{\mathrm{T}} \boldsymbol{F S}\right)^{-1} \boldsymbol{S}^{\mathrm{T}} \boldsymbol{e}_{0}
$$

At the end, the element forces $t$ concerned herein, i.e., the part $\boldsymbol{t}_{\boldsymbol{e}_{0}}$ caused by initial elongations, can be obtained by substituting Eq. (14) into Eq. (12) and ignoring the external loads $f$ :

$$
\boldsymbol{t}=-\boldsymbol{S}\left(\boldsymbol{S}^{\mathrm{T}} \boldsymbol{F} \boldsymbol{S}\right)^{-1} \boldsymbol{S}^{\mathrm{T}} \boldsymbol{e}_{0}=-\boldsymbol{K} \boldsymbol{\Omega} \boldsymbol{e}_{0},
$$

where

$$
\boldsymbol{\Omega}=\boldsymbol{F S}\left(\boldsymbol{S}^{\mathrm{T}} \boldsymbol{F S}\right)^{-1} \boldsymbol{S}^{\mathrm{T}}
$$

Similarly, matrix $\boldsymbol{\Omega}$ is also a scaling matrix that shows the relevance of the element force variation to the initial element elongations. It is easy to prove that the idempotence of matrix $\boldsymbol{\Omega}$, which means the trace of this matrix, is equivalent to its rank, namely, the total degree of static indeterminacy $s$. In view of this, the diagonal entry $\gamma_{i}(i=1,2, \ldots, b)$ of matrix $\boldsymbol{\Omega}$ can be interpreted as the DSI. Moreover, since matrix $\boldsymbol{S}^{\mathrm{T}} \boldsymbol{F S}$ in Eq. (16) is always positive definite, this formula is applicable to either kinematically deter- minate or indeterminate assemblies. It is apparent that the improved formula has a wider range of applicability than the previous one.

\subsubsection{A comparison of both methods}

Two analytical methods for the DSI have been provided, but they are suitable for different ranges. Accordingly, a comparison in the kinematically determinate case (where both methods are valid) is obviously necessary in search for their inherent correlation, i.e., whether both methods are essentially identical. Firstly, as matrix $\boldsymbol{U}_{r_{A}}$ in Eq. (11) is guaranteed to be non-singular under this condition, matrix $\boldsymbol{\Lambda}$ in Eq. (8) can be transformed as

$$
\boldsymbol{\Lambda}=\boldsymbol{I}-\left[\left(\boldsymbol{V}_{r_{A}} \boldsymbol{R}_{1}\right)^{\mathrm{T}} \boldsymbol{K}\left(\boldsymbol{V}_{r_{A}} \boldsymbol{R}_{1}\right)\right]^{-1}\left(\boldsymbol{V}_{r_{A}} \boldsymbol{R}_{1}\right)^{\mathrm{T}} \boldsymbol{K},
$$

where $\boldsymbol{V}_{r_{A}} \boldsymbol{R}_{1}\left(\in \mathbb{R}^{b \times r_{A}}\right)$ is a matrix that comprises the sets of compatible elongations corresponding to the modes of extensional displacements $\boldsymbol{U}_{r_{A}}$. Matrix $\boldsymbol{\Omega}$ in Eq. (16) can be rearranged as

$$
\boldsymbol{\Omega}=(\boldsymbol{F S})\left[(\boldsymbol{F S})^{\mathrm{T}} \boldsymbol{K}(\boldsymbol{F S})\right]^{-1}(\boldsymbol{F S})^{\mathrm{T}} \boldsymbol{K},
$$

where $\boldsymbol{F S}\left(\in \mathbb{R}^{b \times s}\right)$ stands for a matrix that contains the modes of incompatible elongations resulting from the self-stress modes $\boldsymbol{S}$. A comparison between these matrices can be performed by

$$
\begin{aligned}
\boldsymbol{\Omega}-\boldsymbol{\Lambda}= & {\left[\boldsymbol{V}_{r_{A}} \boldsymbol{R}_{1} \boldsymbol{F S}\right]\left(\left[\boldsymbol{V}_{r_{A}} \boldsymbol{R}_{1} \boldsymbol{F S}\right]^{\mathrm{T}}[\boldsymbol{K}]\left[\boldsymbol{V}_{r_{A}} \boldsymbol{R}_{1} \boldsymbol{F S}\right]\right)^{-1} } \\
& {\left[\boldsymbol{V}_{r_{A}} \boldsymbol{R}_{1} \boldsymbol{F S}\right]^{\mathrm{T}}[\boldsymbol{K}]-\boldsymbol{I}=\mathbf{0} . }
\end{aligned}
$$

The above equation manifests that under this condition, matrix $\boldsymbol{\Omega}$ is equal to matrix $\boldsymbol{\Lambda}$. This comparison reveals that in spite of the different formulations, these methods describe the inherent properties of assemblies in the same way. In other words, the results obtained by these formulas are the same. Moreover, as $\left[\boldsymbol{V}_{r_{A}} \boldsymbol{R}_{1} \boldsymbol{F S}\right]_{b \times b}$ in Eq. (19) is a square matrix consisting of element elongations, $\left[\boldsymbol{V}_{r_{A}} \boldsymbol{R}_{1} \boldsymbol{F S}\right]^{\mathrm{T}}$ $\cdot[\boldsymbol{K}]\left[\boldsymbol{V}_{r_{A}} \boldsymbol{R}_{1} \boldsymbol{F S}\right]$ can be interpreted as an elastic energy matrix that accumulates elastic energy in two aspects: $\left(\boldsymbol{V}_{r_{A}} \boldsymbol{R}_{1}\right)^{\mathrm{T}} \boldsymbol{K}\left(\boldsymbol{V}_{r_{A}} \boldsymbol{R}_{1}\right)$ and $(\boldsymbol{F S})^{\mathrm{T}} \boldsymbol{K}(\boldsymbol{F S})$. This finding implies that from the structural standpoint, the initial elongation $\boldsymbol{e}_{0}$ can be equilibrated at the current configuration in two distinct ways: deforming the 
compatible element elongations $\boldsymbol{V}_{r_{4}} \boldsymbol{R}_{1}$ and altering the element forces $\boldsymbol{S}$.

\subsection{Applications of DSI}

According to the definition of the DSI, the sum of all elements' DSI is equal to the total degree of static indeterminacy $s$, showing good agreement with the (modified) Maxwell's rule. This definition suggests that DSI is a basic indicator that reflects the combined effect of the geometry, topology, and stiffness from the standpoint of each element. Notably, it is representative of the symmetric properties; the elements at symmetrical locations are supposed to possess the same DSI value.

In light of the different DSI values, all elements of the given assembly can be grouped into $w$ parts with $c_{k}$ elements in the $k$ th group, which can be adopted as a grouping criterion. To confirm the validity of this criterion, the essence of the DSI will be elucidated from a mathematical viewpoint. For brevity, assuming the diagonal flexibility matrix $\boldsymbol{F}=\boldsymbol{I}_{(b \times b)}$, matrix $\boldsymbol{\Omega}$ in Eq. (16) could be rewritten as

$$
\boldsymbol{\Omega}=\boldsymbol{S}\left(\boldsymbol{S}^{\mathrm{T}} \boldsymbol{S}\right)^{-1} \boldsymbol{S}^{\mathrm{T}}=\boldsymbol{S} \boldsymbol{S}^{\mathrm{T}},
$$

where

$$
\boldsymbol{S}=\left[\begin{array}{cccc}
s_{11} & s_{21} & \cdots & s_{s 1} \\
s_{12} & s_{22} & \cdots & s_{s 2} \\
\vdots & \vdots & & \vdots \\
s_{1 b} & s_{2 b} & \cdots & s_{s b}
\end{array}\right]=\left[\begin{array}{llll}
\tilde{\boldsymbol{s}}_{1} & \tilde{\boldsymbol{s}}_{2} & \cdots & \tilde{\boldsymbol{s}}_{b}
\end{array}\right]^{\mathrm{T}},
$$

where $\tilde{\boldsymbol{s}}_{i}(i=1,2, \ldots, b)$ denotes the $i$ th row vector of matrix $\boldsymbol{S} .\left\|\tilde{\boldsymbol{s}}_{i}\right\|_{2}$ stands for its Euclidean that is characteristic of geometric property. As a result, a new formula for DSI can be expressed in terms of Euclidean norms:

$$
\gamma_{i}=\sum_{j=1}^{s}\left(s_{j i}\right)^{2}=\left(\left\|\tilde{\boldsymbol{s}}_{i}\right\|_{2}\right)^{2}
$$

which conforms to the definition of DSI. It is observed that in accordance with the symmetric properties, the Euclidean norms $\left\|\tilde{\boldsymbol{s}}_{i}\right\|_{2}$ of the elements in one group are identical to each other, regardless of different stiffness assignments. Consequently, these identical Euclidean norms may account for the valid- ity of the proposed criterion, ensuring that symmetrical elements always have the same DSI value.

This grouping criterion using the symmetric properties can be employed into a specific force finding method entitled double singular value decomposition (DSVD) (Yuan et al., 2007). Since the efficiency of the DSVD method is directly related to the means of group division, the proposed criterion guaranteeing a proper grouping proves to be simpler and more efficient, in contrast to the previous criteria that achieve a proper group division by iteratively changing the number of groups (Tran and Lee, 2010; 2013; Tran et al., 2012; Lee et al., 2014). Additionally, it can be inferred that by means of this criterion, specifically, via different stiffness assignments, this improved DSVD method can allow designers more freedom in the initial force design (or force finding).

Apart from the improved DSVD method, the DSI can also be used to demonstrate the influence of the surrounding elements on a specified element. Choosing a single element $i$ that is subjected to an initial elongation $e_{0 i}$, the resulting element force and the elongation are respectively:

$$
\begin{aligned}
& t_{i}=-k_{i} \gamma_{i} e_{0 i}, \quad i=1,2, \cdots, b, \\
& e_{i}^{\prime}=-\gamma_{i} e_{0 i}, \quad i=1,2, \cdots, b,
\end{aligned}
$$

where shortenings $\left(e_{0 i}<0\right)$ create tension, and extensions $\left(e_{0 i}>0\right)$ give rise to compression. For the specified element, its opposite ends are assumed to be completely fixed if the DSI value is equal to one, whereas this element is free to elongate if the value equals zero.

As suggested by prior studies, the DSI is closely connected to the robustness (or vulnerability) analysis as well as structural sensitivity theory. In the context of robustness analyses, the DSI is complementary to the stiffness-based importance index $\lambda$, for example, $\gamma_{i}=1-\lambda_{i}$ for element $i$. This finding means that for a single element, its importance to load-bearing capacity becomes lower as the DSI value increases.

From the perspective of structural sensitivity, the DSI can be applied to evaluate the behaviors of the elements triggered by the initial elongations, such as length imperfections or thermal strains. It is observed from Eq. (8) or Eq. (16) that for an entire assembly, if the DSI of one element is larger than 
that of another, this element is more sensitive to the input of initial elongations. This indicates a new way to determine how accurately a cable-strut structure should be manufactured, in which a greater DSI leads to a higher accuracy requirement for elements.

\section{Distributed kinematic indeterminacy}

As mentioned above, the distributed indeterminacy evaluation of flexible structures considering the effect of component stiffness is discussed here from the aspect of the DSI and that of the DKI. For the concept of the DSI, it has been elaborated in the foregoing section; subsequently, an evaluable method for the DKI will be first proposed and then its interpretation and potential applications will be provided in detail.

\subsection{Mathematical formula for DKI}

In analogy to the unified method for the DSI, the mathematical formulation for the DKI will focus on the relationship between the nodal displacements $\boldsymbol{d}$ and the external loads $\boldsymbol{f}$. In the finite element (FE) method, the external loads $f$ can be written as

$$
\boldsymbol{f}=\boldsymbol{K}_{s} \boldsymbol{d},
$$

where

$$
\boldsymbol{K}_{s}=\boldsymbol{Z}+\boldsymbol{A} \hat{\boldsymbol{G}} \boldsymbol{A}^{\mathrm{T}} .
$$

Matrix $\boldsymbol{K}_{s}$ is the tangent stiffness matrix that consists of the stress matrix (or geometric stiffness matrix) $\boldsymbol{Z}$ and the material stiffness matrix $\boldsymbol{A} \hat{\boldsymbol{G}} \boldsymbol{A}^{\mathrm{T}}$ with $\hat{\boldsymbol{G}}=\operatorname{diag}\left(g_{1}, g_{2}, \ldots, g_{i}, \ldots, g_{b}\right)$, where $g_{i}$ is the modified axial stiffness of element $i$ (Guest, 2006; Sultan, 2013; Zhang et al., 2014). As cable slacking is beyond the scope of this study, matrix $\boldsymbol{Z}$ is presumed to be invertible for simplicity.

Meanwhile, a general expression of nodal displacements $\boldsymbol{d}$ can be expressed as

$$
\boldsymbol{d}=\boldsymbol{B}^{+} \boldsymbol{e}+\boldsymbol{M} \boldsymbol{\beta},
$$

where matrix $\boldsymbol{B}^{+}$is the generalized inverse of matrix $\boldsymbol{B}$ and vector $\boldsymbol{\beta}$ is the coefficient vector of $m$ mechanisms. Assuming that this assembly forbids any kind of element elongation, the nodal displacements $\boldsymbol{d}$ discussed herein are in fact the rigid body displacements and can be reduced to

$$
\boldsymbol{d}=\boldsymbol{M} \boldsymbol{\beta} .
$$

Although the values of vector $\boldsymbol{\beta}$ are arbitrary, for a kinematically indeterminate assembly, a specific vector $\boldsymbol{\beta}$ can be determined by a set of $m$ equilibrium equations. As the external loads $\boldsymbol{f}$ contain two orthogonal portions (Pellegrino and Calladine, 1986), these equilibrium equations can be obtained by

$$
\boldsymbol{M}^{\mathrm{T}}\left(\boldsymbol{f}-\boldsymbol{K}_{s} \boldsymbol{M \beta}\right)=\mathbf{0},
$$

where matrix $\boldsymbol{M}$ mentioned previously can also represent the basic bases for the external loads that cannot be equilibrated at the current configuration, while the expression $\left(\boldsymbol{f}-\boldsymbol{K}_{s} \boldsymbol{M} \boldsymbol{\beta}\right)$ stands for the external loads that can be equilibrated. Since matrix $\boldsymbol{M}$ is the null space of matrix $\boldsymbol{B}=\boldsymbol{A}^{\mathrm{T}}$, substituting Eq. (26) into Eq. (29) and rearranging can yield the coefficient vector $\boldsymbol{\beta}$ as follows:

$$
\boldsymbol{\beta}=\left(\boldsymbol{M}^{\mathrm{T}} \boldsymbol{Z M}\right)^{-1} \boldsymbol{M}^{\mathrm{T}} \boldsymbol{f} .
$$

In the absence of any element elongation, the nodal displacements $\boldsymbol{d}$, i.e., the rigid body displacements, can be achieved by substituting Eq. (30) into Eq. (28):

$$
\boldsymbol{d}=\boldsymbol{M}\left(\boldsymbol{M}^{\mathrm{T}} \boldsymbol{Z} \boldsymbol{M}^{-1}\right) \boldsymbol{M}^{\mathrm{T}} \boldsymbol{f}=\boldsymbol{N} \boldsymbol{\Phi} \boldsymbol{f}
$$

where $\boldsymbol{N}=\boldsymbol{Z}^{-1}$ and

$$
\boldsymbol{\Phi}=\boldsymbol{Z} \boldsymbol{M}\left(\boldsymbol{M}^{\mathrm{T}} \boldsymbol{Z} \boldsymbol{M}^{-1}\right) \boldsymbol{M}^{\mathrm{T}} .
$$

Eq. (31) gives a description about the rigid body movements, where the rigid body displacements are linked by matrix $\boldsymbol{\Phi}$ to the external nodal loads. Since matrix $\boldsymbol{\Phi}$ is an idempotent matrix, the trace of this matrix is equivalent to its rank, namely, the total degree of kinematic indeterminacy $m$. This implies that its diagonal entry can illustrate the contribution of each node to the total kinematic indeterminacy in terms of different directions. Similarly, the diagonal entry $\eta_{j}(j=1,2, \ldots, 3 n-c)$ of matrix $\boldsymbol{\Phi}$ is defined as the DKI. 


\subsection{Applications of DKI}

A conclusion that can be drawn is that because the DKIs of all nodes amount to the total degree of kinematic indeterminacy $m$, the concept of the DKI is consistent with the (modified) Maxwell's rule. This conclusion suggests that as a basic indicator in which the geometry, topology, and geometric stiffness are integrated, the DKI unveils the contribution of each node to the kinematic indeterminacy of an entire assembly in terms of different directions.

In fact, the kinematic indeterminacy studied in this paper corresponds to the degree of freedom $(\mathrm{DoF})$ in kinematics. These findings indicate that the DoF of each node can be assigned to different directions by the DKI. In other words, the DKI can be applied to assess the nodal mobility of an assembly in every equilibrium configuration. The DKI value reveals the possibility of nodal deformation in $x-, y$, or $z$-direction, while the sign represents the relationship between the directions of nodal displacements and external loads. Generally, this application is valid under the scope of small strains and deformations.

However, there is a good chance that with respect to a specified direction, the DKI values of the nodes at symmetrical locations are different. To address this issue, the summation of a node's DKIs $\chi_{h}$ $(h=1,2, \ldots, n)$ can serve as another indicator for the total mobility of each node. The $\chi_{h}$ values of symmetrically located nodes are always the same, irrespective of what coordinate system is chosen to describe the geometry of this assembly.

Additionally, Eq. (31) concerning the rigid body movements controlled by the external loads, can correlate the DKI with the form transforming study of deployable space structures. As stated in the relevant references (Chen et al., 2000; Chen and Zhang, 2006), the structural configuration of every moment can be written as

$$
\boldsymbol{x}(t+\Delta t)=\boldsymbol{x}(t)+\boldsymbol{M} \boldsymbol{\eta}_{0} \boldsymbol{M}^{\mathrm{T}} \boldsymbol{f} \Delta t
$$

where function $\boldsymbol{x}(t)$ depicts the nodal coordinates at the time $t$, vector $\boldsymbol{\eta}_{0}$ contains arbitrary and small coefficients and scalar $\Delta t$ represents the time increment. Note that vector $\boldsymbol{M} \boldsymbol{\eta}_{0} \boldsymbol{M}^{\mathrm{T}} \boldsymbol{f} \Delta t$ can be interpreted as the rigid body displacements during an increment $\Delta t$, while the rigid body displacements $\boldsymbol{d}$ during a unit increment $(\Delta t=1)$ are provided by Eq. (31). Hence, as the definitions of matrix $\boldsymbol{M}$ and vector $\boldsymbol{f}$ remain the same throughout this study, it can be concluded that

$$
\boldsymbol{\eta}_{0}=\boldsymbol{\eta}
$$

where $\boldsymbol{\eta}=\left[\eta_{1}, \eta_{2}, \ldots, \eta_{3 n-c}\right]^{\mathrm{T}}$ and $\eta_{j}$ is the DKI of component $j$. This equation implies that the DKI can provide a suitable vector $\boldsymbol{\eta}_{0}$ for the form transforming study. In addition, the vector $\boldsymbol{\eta}$ formed by the DKI can satisfy the mobility criterion that deployable structures get to a steady state, when the external load vector is orthogonal to the mechanism vector.

\section{Examples}

An algorithm coded by Matlab 2014B is developed for the distributed indeterminacy evaluation as shown in Fig. 1. Three examples of cable-strut structures, including a Geiger dome, a Levy dome, and a tensegrity simplex will be investigated through this algorithm. In these examples, flexible structures are modelled as a set of weightless tensile cables and compressive struts connected by frictionless pin-joints.

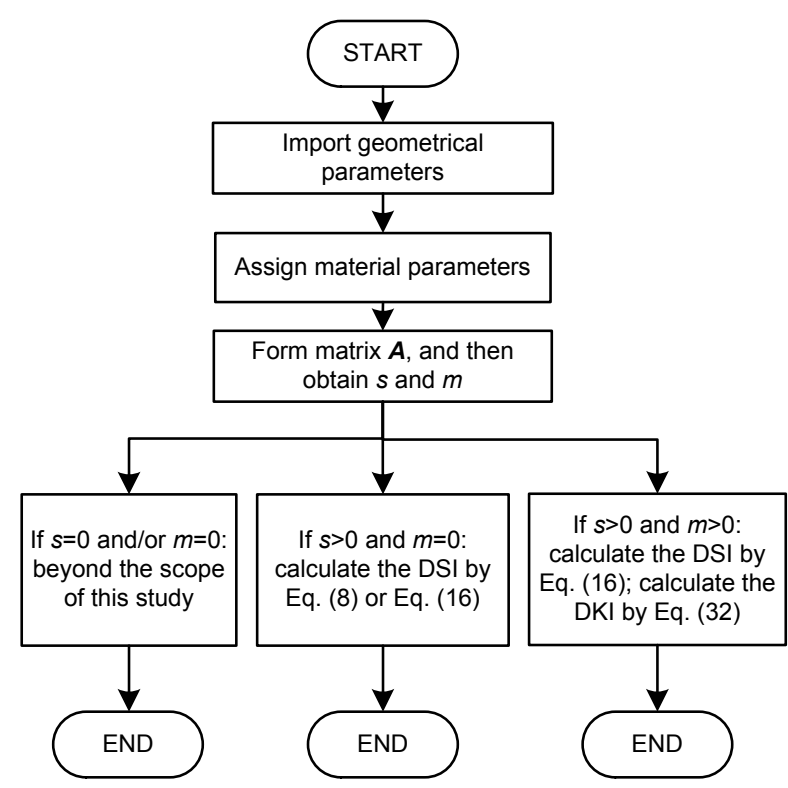

Fig. 1 Flow chart of the algorithm for the distributed indeterminacy evaluation 


\subsection{Example 1}

A Geiger cable dome comprising 49 elements and 26 nodes (eight completely fixed boundary nodes), as illustrated in Fig. 2, is a statically and kinematically indeterminate assembly owning four independent self-stress modes and nine mechanisms. To depict its geometry clearly, a Cartesian coordinate system $x y z$ is shown in Fig. 2a and the material parameters are listed for different kinds of elements in Table 1.

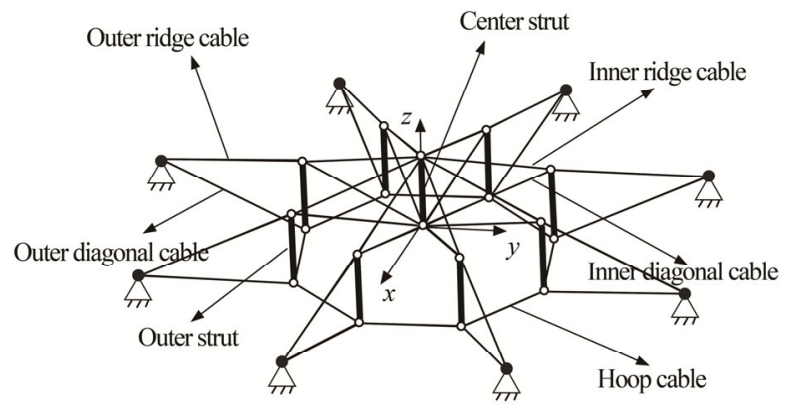

(a)

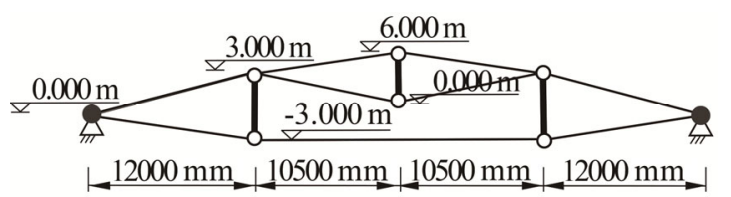

(b)

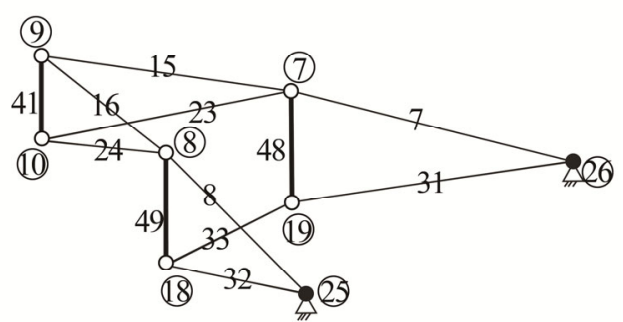

(c)

Fig. 2 Perspective view of struts in thick solid lines (a), section view (b), and an eighth of a Geiger cable dome (c)

At the beginning of this evaluation, the DSI of every element was calculated and is listed in Table 2. The aggregate of these DSIs was equal to the total degree of static indeterminacy $s=4.0000$, which is in good agreement with the (modified) Maxwell's rule. In light of the different DSI values, the given Geiger dome was divided into seven groups (Table 2) corresponding to its symmetric properties. It can be concluded that if the stiffness assignment is changed, another different group division could be gained by means of the new static indeterminacy distribution. Since the DSI is representative of symmetric properties, this grouping criterion can be considered as a simple but efficient way to achieve a proper grouping for the DSVD force finding method.

Furthermore, Table 2 shows that the DSI of the outer ridge cables was 0.2826 , which was larger than those of the others, whereas the DSI of the outer struts was only 0.0002 , which was smaller than the others. This indicates that the outer ridge cables were more sensitive to the imperfections and should be manufactured more accurately, and that as outer struts were of greater importance to the load-bearing capacity, the removal of an outer strut was likely to trigger a larger damage to this assembly.

Table 1 Material parameters of the Geiger cable dome

\begin{tabular}{cc}
\hline Element & $E a(\mathrm{kN})$ \\
\hline Inner ridge cable & 600 \\
Outer ridge cable & 600 \\
Upper hoop cable & 800 \\
Strut & 1000 \\
Inner diagonal cable & 600 \\
Outer diagonal cable & 600 \\
\hline
\end{tabular}

Table 2 DSI of the Geiger cable dome

\begin{tabular}{cccc}
\hline Group & $c_{k}$ & DSI & Member \\
\hline 1 & 1 & 0.0157 & Center strut \\
2 & 8 & 0.0002 & Outer struts \\
3 & 8 & 0.1205 & Inner ridge cables \\
4 & 8 & 0.2826 & Outer ridge cables \\
5 & 8 & 0.0271 & Inner diagonal cables \\
6 & 8 & 0.0380 & Outer diagonal cables \\
7 & 8 & 0.0296 & Hoop cables \\
\hline Total & 49 & 4.0000 & \\
\hline
\end{tabular}

On the other hand, Table 3 presents the DKI and the $\chi_{h}$ values for all nodes of this assembly. As detailed in Table 3, the sum of all DKI values is equal to the degree of kinematic indeterminacy $m=9.0000$; the maxima $\chi_{h}=1.0000$ were at the connection nodes between the inner and the outer ridge 
cables, while the minima $\chi_{h}=0.0000$ were at the opposite ends of the center strut. It can be found that the symmetrically located nodes always have the same $\chi_{h}$ values regardless of the different DKI values. These results suggest that due to the highly latent mobility of these nodes, special attention should be paid to the nodes (7), (8), and those at symmetrical locations.

\subsection{Example 2}

As plotted in Fig. 3, a Levy cable dome is made of 49 elements and 20 nodes; among these nodes, there are six totally restrained boundary nodes. It is assumed that this assembly has the same material

Table 3 DKI of the Geiger cable dome

\begin{tabular}{ccccc}
\hline \multirow{2}{*}{ Node No. } & \multicolumn{3}{c}{ DKI } & \multirow{2}{*}{$\chi_{h}$} \\
\cline { 2 - 4 } 1 & $x-$ & $y-$ & $z-$ & \\
\hline 2 & 0.0000 & 1.0000 & 0.0000 & 1.0000 \\
3 & 0.5000 & 0.5000 & 0.0000 & 1.0000 \\
4 & 1.0000 & 0.0000 & 0.0000 & 1.0000 \\
5 & 0.5000 & 0.5000 & 0.0000 & 1.0000 \\
6 & 0.0000 & 1.0000 & 0.0000 & 1.0000 \\
7 & 0.5000 & 0.5000 & 0.0000 & 1.0000 \\
8 & 1.0000 & 0.0000 & 0.0000 & 1.0000 \\
9 & 0.5000 & 0.5000 & 0.0000 & 1.0000 \\
10 & 0.0000 & 0.0000 & 0.0000 & 0.0000 \\
11 & 0.0000 & 0.0000 & 0.0000 & 0.0000 \\
12 & 0.0000 & 0.1250 & 0.0000 & 0.1250 \\
13 & 0.0625 & 0.0625 & 0.0000 & 0.1250 \\
14 & 0.1250 & 0.0000 & 0.0000 & 0.1250 \\
15 & 0.0625 & 0.0625 & 0.0000 & 0.1250 \\
16 & 0.0000 & 0.1250 & 0.0000 & 0.1250 \\
17 & 0.0625 & 0.0625 & 0.0000 & 0.1250 \\
18 & 0.1250 & 0.0000 & 0.0000 & 0.1250 \\
\hline Total & 0.0625 & 0.0625 & 0.0000 & 0.1250 \\
\hline & & & & 9.0000 \\
\hline
\end{tabular}

parameters as the above Geiger dome. In the current configuration, this assembly was a kinematic determinate assembly having four independent self-stress modes and zero mechanisms.

As a kinematic determinate assembly, the DSI of this assembly can be calculated by two distinct methods as proposed above. The computed DSIs of all elements are detailed in Table 4, the sum of

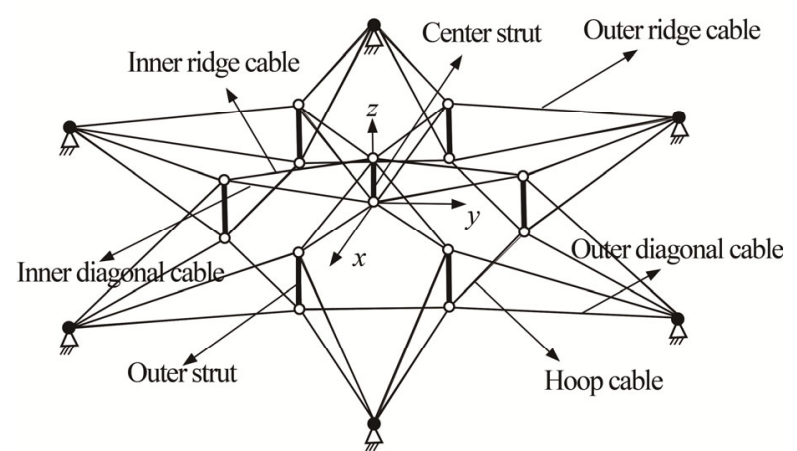

(a)

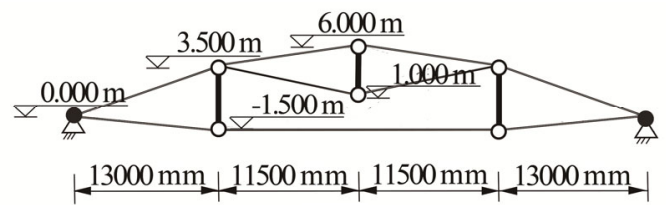

(b)

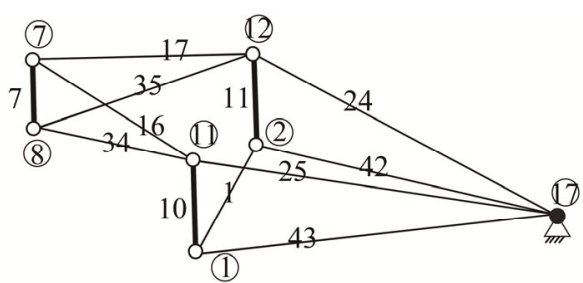

(c)

Fig. 3 Perspective view of struts in thick solid lines (a), section view (b), and a sixth of a Levy cable dome (c)

Table 4 DSI of the Levy cable dome

\begin{tabular}{ccccc}
\hline Group & $c_{k}$ & DSI obtained by Eq. (8) & DSI obtained by Eq. (16) & Member \\
\hline 1 & 1 & 0.0132 & 0.0132 & Center strut \\
2 & 6 & 0.0008 & 0.0008 & Outer struts \\
3 & 12 & 0.0440 & 0.0440 & Inner ridge cables and inner diagonal cables \\
4 & 12 & 0.1212 & 0.1212 & Outer ridge cables \\
5 & 12 & 0.1036 & 0.1036 & Outer diagonal cables \\
6 & 6 & 0.1269 & 0.1269 & Hoop cables \\
\hline Total & 49 & 4.0000 & 4.0000 & \\
\hline
\end{tabular}


which was 4.0000 , i.e., the total degree of static indeterminacy.

As expected, the results of both methods are identical, confirming the inherent correlation between these methods. With these different DSI values, all elements of this assembly can be packed into six groups. Moreover, the maximum value was 0.1269 in the hoop cables, while the minimum was 0.0008 in the outer struts. This finding indicates that the hoop cables are more sensitive to the imperfections and should be manufactured more accurately, and that the outer struts are more important for the load-bearing capability. In comparison to the Geiger dome given above, they had different kinds of the most sensitive components, but the same kind of most important elements (in terms of the loadbearing capability).

\subsection{Example 3}

Fig. 4 illustrates a tensegrity simplex consisting of 16 elements and eight nodes; a Cartesian coordinate system $x y z$ is chosen in Fig. 4a; the top square and the bottom square of this simplex are assembled with a specific twist angle $\beta=45^{\circ}$; the radius of its circumcircle $r$ and the height $h$ are $0.707 \mathrm{~m}$ and $1.000 \mathrm{~m}$, respectively. It was found that this freestanding assembly had one self-stress mode and nine mechanisms containing three internal mechanisms and six rigid body mechanisms.

After eliminating rigid body mechanisms, the DSI and the DKI were calculated and are listed in Tables 5 and 6 , respectively. A similar observation, recorded in both Tables 5 and 6 , was that the sum of DSI values and that of DKI were equal to the degree of static indeterminacy $s=1.0000$ and that of kinematic indeterminacy $m=3.0000$, respectively. As shown in Table 5, this assembly can be classified into three groups by means of these DSIs. Furthermore, elements 13-16 had the largest DSI (0.1367), and elements $1-4$ had the smallest DSI (0.0203). This means that elements $13-16$ were more sensitivity to the imperfections, and that compared with the others, elements 1-4 were relatively important for the load-bearing capacity. In the DKI aspect, all nodes of this assembly owned the same $\chi_{h}=0.3750$, indicating that all nodes had the same mobility no matter what coordinate system had been chosen to depict its geometry.

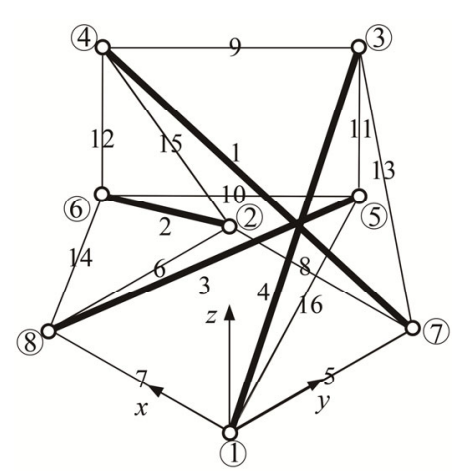

(a)

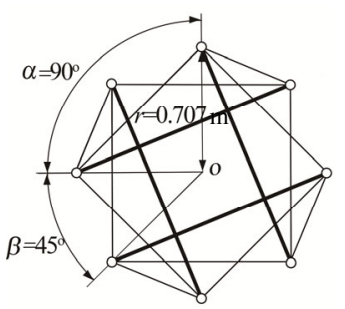

(b)

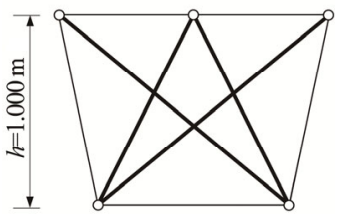

(c)
Fig. 4 A tensegrity simplex with struts in thick solid lines (a), top view, where point $O$ is the midpoint of the square (b), and side view (c)

Table 5 DSI of the tensegrity simplex

\begin{tabular}{cccc}
\hline Group & $c_{k}$ & DSI & Member \\
\hline 1 & 4 & 0.0203 & $1-4$ \\
2 & 8 & 0.0465 & $5-12$ \\
3 & 4 & 0.1367 & $13-16$ \\
\hline Total & 16 & 1.0000 & \\
\hline
\end{tabular}

Table 6 DKI of the tensegrity simplex

\begin{tabular}{|c|c|c|c|c|}
\hline \multirow{2}{*}{ Node No. } & \multicolumn{3}{|c|}{ DKI } & \multirow{2}{*}{$\chi_{h}$} \\
\hline & $x-$ & $y$ - & $z-$ & \\
\hline 1 & 0.8955 & -1.1898 & 0.6693 & 0.3750 \\
\hline 2 & 0.8955 & -1.1898 & 0.6693 & 0.3750 \\
\hline 3 & -0.4314 & 0.1371 & 0.6693 & 0.3750 \\
\hline 4 & 0.1371 & -0.4314 & 0.6693 & 0.3750 \\
\hline 5 & 0.1371 & -0.4314 & 0.6693 & 0.3750 \\
\hline 6 & -0.4314 & 0.1371 & 0.6693 & 0.3750 \\
\hline 7 & -1.1898 & 0.8955 & 0.6693 & 0.3750 \\
\hline 8 & -1.1898 & 0.8955 & 0.6693 & 0.3750 \\
\hline Total & & & & 3.0000 \\
\hline
\end{tabular}

\section{Conclusions}

This paper is mainly concerned with distributed indeterminacy evaluation including the DSI and the DKI; its key objective is to provide reasonable interpretations and feasible applications in engineering.

A unified method for the DSI was proposed and then a comparative study between it and an existing method was conducted. Unlike the existing method, 
the proposed one avoids the problems of singularity and therefore has a wider range of applicability, which means this method is applicable to either kinematically determinate or indeterminate assemblies. This comparison also reveals that despite the fact that these methods are suitable for different ranges, they are essentially identical in the kinematically determinate case.

As a basic indicator of structural properties, the DSI can be used to assess the structural sensitivity as well the robustness (or vulnerability). Most notably, since the DSI is representative of symmetric properties, a simple but efficient grouping criterion can be established in the light of the DSI, which can improve the efficiency of the DSVD force finding method and allow more freedom for designers in the initial force design.

Another important aspect of this study is that to the best of our knowledge this is the first study to establish an evaluable method for the DKI, i.e., the complementary concept of the DSI. This method indicates that the DKI is a useful indicator for the assessment of nodal mobility and can propose a feasible solution to the form transforming study.

\section{References}

Ashwear, N., Eriksson, A., 2014. Natural frequencies describe the pre-stress in tensegrity structures. Computers \& Structures, 138:162-171. [doi:10.1016/j.compstruc.2014. 01.020]

Calladine, C., 1978. Buckminster Fuller's "tensegrity" structures and Clerk Maxwell's rules for the construction of stiff frames. International Journal of Solids and Structures, 14(2):161-172. [doi:10.1016/0020-7683(78)900525]

Calladine, C., Pellegrino, S., 1991. First-order infinitesimal mechanisms. International Journal of Solids and Structures, 27(4):505-515. [doi:10.1016/0020-7683(91)901375]

Chen, Q., Kou, X.J., 2013. A constraint matrix approach for structural ultimate resistance to access the importance coefficient values of rigid joints. Advances in Structural Engineering, 16(11):1863-1870. [doi:10.1260/1369-4332. 16.11.1863]

Chen, Q., Kou, X.J., Zhang, Y., 2010. Internal force and deformation matrixes and their applications in load path. Journal of Zhejiang University-SCIENCE A (Applied Physics \& Engineering), 11(8):563-570. [doi:10.1631/ jzus.A0900630]

Chen, W.J., Zhang, S.J., 2006. Deployable Space Structures and Analysis Theory. China Astronautic Publishing House, Beijing, China (in Chinese).
Chen, W.J., Guan, F.L., Dong, S.L., 2000. Dynamic analysis of deployable space truss structures. Chinese Journal of Computational Mechanics, 17:411-416 (in Chinese).

Chen, W.J., Zhou, J.Y., Zhao, J.Z., 2014. Computational methods for the zero-stress state and the pre-stress state of tensile cable-net structures. Journal of Zhejiang University-SCIENCE A (Applied Physics \& Engineering), 15(10):813-828. [doi:10.1631/jzus.A1400080]

Eriksson, A., Tibert, A.G., 2006. Redundant and forcedifferentiated systems in engineering and nature. Computer Methods in Applied Mechanics and Engineering, 195(41-43):5437-5453. [doi:10.1016/j.cma.2005.11.007]

Gao, Y., Liu, X.L., 2013. Weighted graph form of structures and its application in robustness analysis. Journal of Shanghai Jiaotong University (Science), 18(2):216-223. [doi:10.1007/s12204-013-1385-2]

Guest, S., 2006. The stiffness of prestressed frameworks: a unifying approach. International Journal of Solids and Structures, 43(3-4):842-854. [doi:10.1016/j.ijsolstr.2005. 03.008]

Jiang, M., Kou, X.J., Li, Z.M., 2012. The redundancy matrix of rigid-frame structure and its application. Journal of Donghua University, 29:107-110.

Kou, X.J., Chen, Q., Song, J., 2008. Reliability estimation involving indirect load effects. Proceeding of the 4th Asian-Pacific Symposium, Hong Kong, p.137-140.

Lee, S., Woo, B., Lee, J., 2014. Self-stress design of tensegrity grid structures using genetic algorithm. International Journal of Mechanical Sciences, 79:38-46. [doi:10.1016/ j.ijmecsci.2013.12.001]

Pai, P.F., 2011. Three kinematic representations for modeling of highly flexible beams and their applications. International Journal of Solids and Structures, 48(19):27642777. [doi:10.1016/j.ijsolstr.2011.06.001]

Pellegrino, S., 1990. Analysis of prestressed mechanisms. International Journal of Solids and Structures, 26(12): 1329-1350. [doi:10.1016/0020-7683(90)90082-7]

Pellegrino, S., 1993. Structural computations with the singular value decomposition of the equilibrium matrix. International Journal of Solids and Structures, 30(21):30253035. [doi:10.1016/0020-7683(93)90210-X]

Pellegrino, S., Calladine, C.R., 1986. Matrix analysis of statically and kinematically indeterminate frameworks. International Journal of Solids and Structures, 22(4):409428. [doi:10.1016/0020-7683(86)90014-4]

Ströbel, D., 1995. Die Anwendung der Ausgleichungsrechnung auf Elastomechanische Systeme. PhD Thesis, Universität Stuttgart, Stuttgart, Germany (in German).

Ströbel, D., Singer, P., 2008. Recent developments in the computational modelling of textile membranes and inflatable structures. In: Oñate, E., Kröplin, B. (Eds.), Textile Composites and Inflatable Structures II. Springer Netherlands, p.253-266. [doi:10.1007/978-1-4020-6856-0_14]

Sultan, C., 2013. Stiffness formulations and necessary and sufficient conditions for exponential stability of prestressable structures. International Journal of Solids and 
Structures, 50(14-15):2180-2195. [doi:10.1016/j.ijsolstr. 2013.03.005]

Tibert, A.G., 2005a. Distributed indeterminacy in frameworks. Proceedings of the 5th International Conference on Computation of Shell and Spatial Structures, Salzburg, Austria.

Tibert, A.G., 2005b. Flexibility evaluation of prestressed kinematically indeterminate frameworks. The 18th Nordic Seminar on Computational Mechanics, Helsinki, Finland.

Tran, H.C., Lee, J., 2010. Initial self-stress design of tensegrity grid structures. Computers \& Structures, 88(9-10): 558-566. [doi:10.1016/j.compstruc.2010.01.011]

Tran, H.C., Lee, J., 2013. Form-finding of tensegrity structures using double singular value decomposition. Engineering with Computers, 29(1):71-86. [doi:10.1007/ s00366-011-0245-7]

Tran, H.C., Park, H.S., Lee, J., 2012. A unique feasible mode of prestress design for cable domes. Finite Elements in Analysis and Design, 59:44-54. [doi:10.1016/j.finel. 2012.05.004]

Yuan, X.F., Chen, L.M., Dong, S.L., 2007. Prestress design of cable domes with new forms. International Journal of Solids and Structures, 44(9):2773-2782. [doi:10.1016/j. ijsolstr.2006.08.026]

Zhang, L.Y., Li, Y., Cao, Y.P., et al., 2013. A unified solution for self-equilibrium and super-stability of rhombic truncated regular polyhedral tensegrities. International Journal of Solids and Structures, 50(1):234-245. [doi:10. 1016/j.ijsolstr.2012.09.024]

Zhang, L.Y., Li, Y., Cao, Y.P., et al., 2014. Stiffness matrix based form-finding method of tensegrity structures. Engineering Structures, 58:36-48. [doi:10.1016/j.engstruct. 2013.10.014]

\section{中文概要}

题 目: 索杆结构分布式不定数分析: 推导和应用

目 的: 针对预张力索杆体系, 将构件刚度与体系判定 相结合, 提出分布式静不定和分布式动不定的 计算方法, 使体系分析从 “系统” 层面向 “构 件”层面延伸。

创新点: 1. 推导出具有广泛适应性的分布式静不定公 式, 并证明与原有方法的内在关系。2. 首次提 出分布式动不定数学公式。3. 给出分布式不定 数的物理意义及潜在的应用。

方 法: 该方法在平衡矩阵理论基础上, 采用奇异值分 解法分别求解相互正交的两类单元变形量和两 类节点外荷载模态; 在排除整体刚体位移模态 后, 利用该正交性, 求解分布式静不定和动 不定。

结 论: 1. 该方法能克服已有方法中的奇异性问题, 具 有普遍性, 可适用于动定及动不定结构。2. 作 为结构双对称性的代表, 分布式静不定数可被 用作一个简单而有效的分组准则; 该准则能提 高二次奇异值找力法 (DSVD) 的效率并能为设 计师提供更多的初始预应力设计可能性。3. 揭 示分布式静不定与结构重要性及结构敏感性间 的关系。4. 分布式动不定数可被用作节点可动 性的一个基本指标。

关键词: 柔性结构; 索杆结构; 分布式不定数; 初始预 应力设计; 找力分析; 奇异值分解; 形状变换 